Editorial

\title{
Acknowledgement to Reviewers of JCM in 2018
}

\author{
JCM Editorial Office \\ MDPI, St. Alban-Anlage 66, 4052 Basel, Switzerland; jcm@mdpi.com \\ Published: 8 January 2019
}

Rigorous peer-review is the corner-stone of high-quality academic publishing. The editorial team greatly appreciates the reviewers who contributed their knowledge and expertise to the journal's editorial process over the past 12 months. In 2018, a total of 588 papers were published in the journal, with a median time to first decision of 15 days and a median time to publication of 31 days. The editors would like to express their sincere gratitude to the following reviewers for their cooperation and dedication in 2018:

\begin{tabular}{ll} 
Aagaard, Niels K. & Ashton, James \\
Abbas-Aghababazadeh, & Assenat, Eric \\
Farnoosh & Avadhanula, Vasanthi \\
Abdul Salim, Sohail & Aveline, Christophe \\
Abe, Masanori & Azuma, Toshifumi \\
Abete, Pasquale & Backer, Daniel De \\
Abuzaid, Ahmed & Badlissi, Fadi \\
Addy, Charlotte & Balasubramanian, Ganesh \\
Ahmed, Naseer & Ballesteros, Soledad \\
Airagnes, Guillaume & Balouch, Sara \\
Akamizu, Takashi & Banach, Maciej \\
Alakhras, Maram & Bancos, Irina \\
Al-Bahrani, Reda & Bang, Marie-Louise \\
Albert, Stewart & Baniahmad, Aria \\
Alesi, Marianna & Banyasz, Alissa M. \\
Aloisi, Anna M. & Baracos, Vickie \\
Al-Samkari, Hanny & Baragetti, Andrea \\
Alvarez-Llamas, Gloria & Baran, Robert \\
Ammer, Kurt & Barchetta, Ilaria \\
Amole, Morolake & Barichello, Tatiana \\
Anadol, Remzi & Barranco Ruiz, Yaira \\
Anderson, Craig & Barratt, Shaney \\
Andersson, Karl & Barreto, Erin F. \\
Ansani, Lucia & Barton, Matthew J. \\
Antunes, Hugo & Bastian, Max \\
Anurag, Meenakshi & Batinic-Haberle, Ines \\
Araújo, João Ricardo & Bauer, Corinna \\
Arcila, Maria E. & Bauer, Stuart B. \\
Arentson-Lantz, Emily & Baydur, Ahmet \\
Armando, Stefanati & Beaumont, Robin \\
Arnaout, Bachaar & Beckmann, Manfred \\
Artioli, Grazia & Beer, Ambros J. \\
Artunc, Ferruh & Beeri, Michal S. \\
Arveschoug, Anne K. & Behrendt, Christian A. \\
Asatryan, Babken & Beisswenger, Christoph \\
& \\
\hline &
\end{tabular}

Aagaard, Niels K.

Abbas-Aghababazadeh,

Abdul Salim, Sohail

Abe, Masanori

Abete, Pasquale

Abuzaid, Ahmed

Addy, Charlotte

Ahmed, Naseer

Airagnes, Guillaume

Akamizu, Takashi

Alakhras, Maram

Al-Bahrani, Reda

Albert, Stewart

Al-Samkari, Hanny

Alvarez-Llamas, Gloria

Ammer, Kurt

Anadol, Remzi

Anderson, Craig

Antunes, Hugo

Anurag, Meenakshi

Araújo, João Ricardo

Arcila, Maria E.

Armando, Stefanati

Arnaout, Bachaar

Artioli, Grazia

Arveschoug, Anne K.

Asatryan, Babken
Ashton, James

Aveline, Christophe

Azuma, Toshifumi

Badlissi, Fadi

Balasubramanian, Ganesh

Ballesteros, Soledad

Balouch, Sara

Banach, Maciej

Bancos, Irina

Bang, Marie-Louise

Baniahmad, Aria

Banyasz, Alissa M.

Baracos, Vickie

Baragetti, Andrea

Baran, Robert

Barchetta, Ilaria

Barichello, Tatiana

Barratt, Shaney

Bastian, Max

Batinic-Haberle, Ines

Bauer, Corinna

Beaumont, Robin

Beckmann, Manfred

Beer, Ambros J.

Beeri, Michal S.

Beisswenger, Christoph
Barreto, Erin F.
Bejjanki, Harini

Berger, Mette

Bermejo-Martin, Jesús F.

Bernat, James L.

Bernstein, Hans-Gert

Berveiller, Paul

Bhutta, Mahmood

Bijlsma, Maarten J.

Bikov, András

Birchard, Thaddeus

Biruete, Annabel

Bishwajit, Ghose

Boentert, Matthias

Boezeman, Edwin J.

Borghi, Claudio

Bosman, Giel

Bottiger, Ylva

Bowling, April

Braat, Andries E.

Bradley, Elyse C.

Brand, Serge

Brazo, Perrine

Briassoulis, George

Brietzke, Scott E.

Brochiero, Emmanuelle

Brown, Joshua D.

Bruins Slot, Karsten

Bryniarski, Krzysztof

Buckley, Nicholas

Buell, Thomas J.

Bujan-Rivas, Segundo

Bukhari, Marwan A.S.

Bulum, Tomislav

Burjek, Nicholas E.

Burkard, Thilo 
Burr, Lucy

Bush, Douglas

Buxi, Dilpreet

Cadario, Francesco

Caironi, Pietro

Calabro, Rocco Salvatore

Calle Rubio, Myriam

Cammu, Guy

Cappariello, Alfredo

Carella, Angelo M.

Caretti, Anna

Carulli, Christian

Casans Francés, Rubén

Casarini, Livio

Casella, Michela

Castagnini, Francesco

Casucci, Gerardo

Cazzador, Diego

Cetin, Irene

Chacón-Borrego, Fátima

Chamaria, Surbhi

Chang, Ke-Vin

Chappel, Eric

Chehroudi, Babak

Chen, Kuo-Hu

Chen, Yun-Ju

Chhabra, Saurabh

Chiang, Tai-An

Chiappori, Alberto A.

Childs, Jessie T.

Chin, Paul

Chiu, Yu-Han

Chmurzyńska, Agata

Chou, Angela

Choudhury, Devasmita

Chue, Pierre S.

Cicero, Arrigo

Citterio, Davide

Clark, Edward

Clouston, Sean A.P.

Coccheri, Sergio

Coffey, Robert J.

Cohen, Steven M.

Cooper, Simon

Corr, Philip

Correale, Michele

Cortese, Antonio

Cortese, Francesca

Costa, Franceso

Cote, Patrice

Coulter, Jonathan A.

Crescioli, Clara
Crisafulli, Ernesto

Crosbie-Watson, Rachelle

Crowley, Jennifer

Crowley Jr., William F.

Cunha, Rodrigo

Cuspidi, Cesare

Cybulski, Mateusz

Damalanka, Vishnu

Danner, Simon M.

Darcy, Justin

Dasari, Surendra

Datta, Poulami

Daubert, Diane M.

De Hair, Maria

De La Iglesia-Garcia, Daniel

De Lucia, Orazio

De Nicola, Luca

De Pinto, Mario

Dedy, Nicolas J.

Degens, Hans

Del Bono, Valerio

Demidem, Aicha

Deraco, Marcello

Deschamps, Jean

Detloff, Megan Ryan

Detti, Laura

D'Ettorre, Gabriele

Dev Jayant, Rahul

Devy, Zisman

Dhanekula, Raja Koteswar

Di Mauro, Michele

Di Stefano, Antonio

Dias, Irundika H.K.

Didehbani, Nyaz

DiMeglio, Linda A.

Dionisi, Francesco

Dissanayake, Hasthi

Djukanović, Ljubica

Donadio, Carlo

Donofry, Shannon D.

Dontje, Manon

Doulias, Paschalis-Thomas

Dowland, Samson N.

Druey, Kirk M.

Duann, Jeng-Ren

Dublin, Sascha

Dudakova, Lubica

Dullaart, Robin P.F.

Dumitrascu, Dan

Dumitrascu, Traian

Dusso, Adriana

Dutta, Sunil
Dwidmuthe, Samir

Eaton, William

Ekúndayò, Olúgbémiga T.

Elson, Daniel

Emilsson, Louise

Enomoto, Hirayuki

Esteve-Puig, Rosaura

Evangelista, Laura

Everaert, Karel

Facchin, Federica

Facchinetti, Fabio

Farber, Harrison W.

Farrell, Stephen W.

Fawkner, Samantha G.

Feldman, Klara R.

Ferrie, Suzie

Finn, Aloke V.

Fischli, Stefan

Fliedner, Stephanie

Floros, Georgios

Forget, Patrice

Formenti, Federico

Fortunato, Orazio

Fragkos, Konstantinos

Francesco, Locatelli

Francois, Patrice

Frankova, Sona

Franzè, Annamaria

Fraser, Steve F.

Fredriksson-Larsson, Ulla

Frisk, Gun

Frohman, Michael

Frykholm, Peter

Fuelesdi, Bela

Fujita, Kohei

Fülöp, Tibor

Fumagalli, Francesca

Fürnsinn, Clemens

Gaitskell, Kezia

Galbán, Craig J.

Gama, Vivian

Gandhi, Shashank V.

García Vela, José A.

Garg, Lohit

Gatla, Himavanth

Gaudino, Mario

Geisler, Corinna

Gentilini, Fabio

Ghisletta, Paolo

Gholami, Sepideh

Giaconi, Jo Ann A.

Giannoni, Patrizia 
Gifford, Paul

Gillies, Glenda

Gitelman, Stephen E.

Giulivi, Cecilia

Giusti, Anna M.

Glasoe, Ward M.

Glueck, Charles J.

Gluud, Lise

Gnambs, Timo

Gnanamony, Manu

Gold, Jason S.

Golding, Jean

Goldsmith, David

Golestaneh, Ladan

Goodman, Annekathryn

Goodyear, Victoria

Gordon, Eliza L.

Gorelik, Oleg

Gorringe, Kylie

Goulielmos, George N.

Gouvernet, Brice

Grabowski, Marcin

Gråstén, Arto

Grattagliano, Ignazio

Graudins, Linda V.

Graves, Jennifer S.

Greupink, Rick

Grimaldi, Domenico

Gris, Jean C.

Grond-Ginsbach, Caspar

Guedeney, Paul

Gugatschka, Markus

Guru, Pramod

Hackett, Geoff

Hadjadj, Samy

Hafer, Carsten

Hage, Camilla

Hagner-Derengowska,

Magdalena

Hall, Shana A.

Hall, Stephanie E.

Hamdy, Reggie C.

Haneke, Eckart

Hannafon, Bethany

Hans, Guy

Hansen, Solveig Lena

Hanudel, Mark

Harari, Sergio

Harati, Ali

Harauz, George

Harcourt, Brooke

Häring, Hans-Ulrich
Haron, Mona

Hartley, Caroline

Hartman, John D.

Hartmann, Hagen

Hautz, Theresa

Heads, Angela M.

Hecker, Andreas

Hedberg, Pär

Hedhammar, My

Heldner, Mirjam

Helliwell, Philip S.

Heo, Dae Seog

Hes, Frederik J.

Hess, Jochen

Heyn, Patricia

Hicks, Alexander

Hideaki, Soya

Hill, Aileen

Hilton, Claudia

Hirahara, Noriyuki

Hochberg, Irit

Hoff, Paula

Hoffman, Robert

Homko, Carol J.

Hoppe, Liesa Katharina

Hori, Hikaru

Hoyer, Erik H.

$\mathrm{Hu}$, Bohua

Huang, Longshuang

Huang, Shiang-Fen

Huang, Shih-Yi

Hughson, Michael D.

Huh, Jin Won

Hwang, Jungyun

Hyman, James M.

Igarashi, Kazuei

Iglesias, Juan F.

Igloi, Kinga

Iliou, Marie C.

Imai, Takao

Imfeld, Patrick

Ingenbleek, Yves

Ino, Yasushi

Inokuchi, Wataru

Iorio, Anna L.

Iorio, Raffaele

Itamar, Raz

Ito, Shigeki

Izumi, Kouji

Jaberi, Aala

Jacobs, David

Jaekel, Julia
Jaimes, Edgar A.

Jain, Hana

Jalava, Katri

Jayant, Kumar

Johnson, Stuart

Jorsäter-Blomgren, Kerstin

Jost, Gregory F.

Juhasz, Bela

Jules, Kevin

Júlíusson, Pétur Benedikt

Kadambi, Pradeep V.

Kakinuma, Yoshihiko

Kalfert, David

Kallonen, Teemu

Kalra, Sanjog

Kalwat, Michael A.

Kamijo-Ikemori, Atsuko

Kamiya, Hideki

Kampmann, Ulla

Kang, Inhae

Kantrowitz, Joshua

Kanzow, Philipp

Karakochuk, Crystal

Karan, Radmila

Karlsson, Hakan

Kaskel, Frederick

Kaushik, Ashlesha

Kawulok, Michal

Kazemi-Bajestani, Seyyed

Mohammad Reza

Kazuhiko, Tsuruya

Keane, David

Keane, Kevin

Keenan, Michael

Keklikoglou, Ioanna

Kelz, Max

Kennedy, Mary-Claire

Kennedy, Norelee

Kern, Ben

Khoury, Samar

Kidd, Timothy J.

Kim, Bhumsoo

Kim, Jennifer A.

Kindermann, Harald

Kinoshita, Akiyoshi

Kirwan, Chris

Knohl, Stephen J.

Knott, Fiona

Kobler, James

Kodama, Daisuke

Koga, Fumitaka

Kolen, Angela M. 
Konialis, Christopher

Konstantinidou, Maria K.

Koshiyama, Masafumi

Kosmidou, Ioanna

Kotsaki, Antigoni

Krafft, Christoph

Krause, Irit

Kroiss, Alexander

Kropp, Martina

Kruger, Tillmann H.C.

Krupinski, Elizabeth

Kukshal, Vandna

Kullberg, Bart-Jan

Kumar, Amit

Kumar, Jayant

Künzel, Julian

Kuperman, Ethan F.

Kuźma-Kozakiewicz,

Magdalena

Kuzmiene, Loreta

Kwiatkowski, David

LaMarca, Babette

Langabeer, James R.

Lappin, Terry

Larange, Alexandre

Leach, Steven T.

Lee, Minsun

Lee, Taikjin

Leger, Luc

Lehrer, Paul

Lemoine, Marie-Eve Lemoine

Lempereur, Mathieu

Lenders, Jacques

Leonetti, Carlo

Lewald, Heidrun

Li, Chia-Jung

Li, Hong-Zhe

Li, Shu-Zhao

Liabeuf, Sophie

Liamis, George

Liao, Ai-Ho

Licari, Amelia

Lichtenstein, Mia B.

Liehn, Elisa A.

Lillehoj, Erik

Lin, Chih-Peng

Lin, Rui-Ting

Lindner, Philip

Lingappan, Krithika

Link, Alexander

Lipnicki, Darren

Lippert-Rasmussen, Kasper
Lipson, Michael J.

Littlefield, Andrew

Livraghi-Butrico, Alessandra

Logan, Sreemathi

Lomero, Marta Gómara

Long, Elizabeth

Lopez-Campos, Jose Luis

López-López, José

Lopomo, Nicola F.

Louch, William E.

Luo, Hua-Cheng

Lupien, Andréanne

Lutz, Wolfgang

Lynch, Christopher

Ma, Xing-Jun

Maas, Renke

MacMillan, Freya

Magenes, Giovanni

Magni, Paolo

Malik, Afshan

Malone, Daniel C.

Mamas, Thalia

Mañago, Mark M.

Mandigout, Stéphane

Mang, Thomas S.

Männikkö, Minna

Mansky, Kim C.

Mantadakis, Elpis

Manzano, Luis

Manzano, Raquel

Marc, Miravitlles

Margulis, Andrea V.

Marozas, Vaidotas

Marra, Paolo

Marteau, Philippe

Martínez-Pinilla, Eva

Martinez-Salgado, Carlos

Marverti, Gaetano

Massa, Horace

Matsunaga, Kazuhisa

Matsuzaki, Junichi

Mattace-Raso, Francesco U.S.

Mattan, Bradley D.

Matteoli, Sara

Mattsson, Janet

McGregor, Gordon

McHugh, Neil

McKenna, Peter E.

McLoney, Eric D.

McPhee, Jamie

McWilliams, Daniel F.

Mearelli, Filippo
Meijers, Björn

Mekontso Dessap, Armand

Melkani, Girish

Mellemkjaer, Lene

Mercieri, Marco

Mete, Ozgur

Michálek, Pavel

Migliore, Federico

Milanaik, Ruth Lynn

Mills, Jesse N.

Milone, Marco

Min, Sedong

Misra, Stuti

Mitchell, Lana

Mittal, Gayatri

Mizuta, Kentaro

Mocellin, Simone

Mochizuki, Naoki

Moen, Aina E.F.

Mohammed, Manal

Molins, Claudia R.

Mollace, Vincenzo

Montrucchio, Giuseppe

Morata, Laura

Morell, Ferran

Mori, Hirotada

Mori, Kiyoshi

Morres, Ioannis

Moustakas, Aristidis

Müller-Myhsok, Bertram

Murakoshi, Nobuyuki

Murphy, Miles A.

Myers, Mark

Nachabé, Rami A.

Nagpal, Seema

Nagulapalli Venkata, Kalyan

Naka, Hiroaki

Napoli, Angela

Naranjo-Hernández, David

Nardi, Paolo

Naruse, Mitsuhide

Natarajan, Arutselvan

Nathan, Cherie-Ann O.

Nauck, Michael

Neri, Matteo

Nestor, Casey

Newell-Fugate, Annie E.

Nicholas, Susanne B.

Nicola, Fusco

Nicolau, David

Nicoletti, Ferdinando

Nierkens, Stefan 
Niikura, Ryota

Nikoletou, Dimitra

Nishikawa, Tetsuo

Niyazov, Dmitriy M.

Noiri, Eisei

Nomellini, Vanessa

Nouri, Aria

Nowak, Elizabeth C.

Nowrouzi-Kia, Behdin

Nugter, Annet

Nunez, Desmond A.

Nurwidya, Fariz

Nyende, Hawa

Oancea, Cristian

O'Neal, Hollis

Ooi, Kenneth

Ørngreen, Mette Cathrine

Ortega-Loayza, Alex

Ortiz Arduan, Alberto

Oudman, Erik

Ozen, Gulsen

Pace, Fabio

Padala, Kalpana P.

Padmanabhan, Jagannath

Paduch, Roman

Pagel, James F.

Paine, Ananta

Palladino, Raffaele

Pan, Zhu-Jun

Panahiazar, Maryam

Papadopoulou, Alexandra

Paparella, Domenico

Papazoglou, Konstantinos

Park, Kwang B.

Parsons, Barbara

Pascual, Julio

Pasquel, Francisco

Pastori, Daniele

Patron, Roberto L.

Patschan, Oliver

Pavarin, Raimondo M.

Pawlitzki, Marc

Pedergnana, Vincent

Pedro, Maria T.

Peinado, Juan R.

Peitsidis, Panagiotis

Pellicori, Pierpaolo

Peng, Robert

Perego, Carla

Pérez-López, Alberto

Pérez-Vázquez, Paz

Perkisas, Stany
Perloff, Michael D.

Persani, Luca

Peter, Lukáš

Phillips, Mary E.

Piepmeier, Aaron

Pina, Melanie

Plasqui, Guy

Polak, Sebastian

Polasek, Thomas M.

Poldermans, Don

Polguj, Michal

Poli, Giuseppe

Pomeroy, Jeremy

Pontrelli, Paola

Postuła, Marek

Preciado, Diego

Prêle, Cecilia

Prior, Steven J.

Priyamvada, Lalita

Quencer, Keith

Quigley, Eamonn

R. Shaker, Mohammed

Rachek, Lyudmila I.

Rago, Anna

Rall, Glenn F.

Rambhatla, Amarnath

Ramot, Yuval

Rasuli, Pasteur

Raw, Rachael K.

Reichert, David E.

Reimers, Anne K.

Reiner, Anne S.

Reiser, Jochen

Reisfeld, Sharon

Remuzzi, Giuseppe

Reschke, Millard F.

Reynolds, Katharine C.

Reznikov, Leah R.

Richards, Jeremy B.

Rimaitis, Kęstutis

Rivas, Carol A.

Rivera, Eleanor

Rizvi, Ali A.

Rizzo, Giuseppe

Robert, Battat

Roberts, Drucilla J.

Robinson, Edward

Robinson, Philip

Roeser, Mark

Roilides, Emmanuel

Rojas-Peña, Alvaro

Romo, Jesus
Ronco, Pierre

Ronellenfitsch, Ulrich

Roper, Randall J.

Rosaly, Correa-de-Araujo

Rosenfield, Mark

Rosenzweig, Derek

Rossi, Elio

Rothe, Hansjörg

Rouse, Rodney

Roviello, Giandomenico

Rudrappa, Mohan

Ruppen, Wilhelm

Rutegård, Martin

Rzońca, Ewa

Sacerdote, Paola

Saintigny, Pierre

Sakhaee, Khashayar

Sakurada, Tsutomu

Salahudeen, Mohammed Saji

Saleh, Tareq

Salgarelli, Attilio C.

Salinas, Jennifer

Salmasi, Amirali

Salminen, Paulina

Sambri, Vittorio

Samuel, Stuart

Sánchez, Mikel

Sancho, Jaime

Sandblom, Gabriel

Sands, Tristan T.

Santos, Luis

Santosa, Hendrik

Sapey, Elizabeth

Sasahira, Tomonori

Saulacic, Nikola

Savarino, Vincenzo

Savastano, Silvia

Schattman, Glenn L.

Schernthaner, Gerit-Holger

Scherrer, Jeffrey F.

Schiano, Concetta

Schob, Stefan

Schreckenberger, Mathias

Schumacher, Udo

Searle, Amelia K.

Sebastian, Ludyga

Selva-O'Callaghan, Albert

Senesi, Pamela

Sessa, Aurelio

Shapiro, Charles L.

Sharma, Aditya

Sharma, Isha 
Sharma, Pawan

Sharma, Ritin

Sharpe, Louise

Shaw, George J.

Sherratt, Simon

Shi, Yi

Shiga, Kiyoto

Shirotsuki, Kentaro

Shiu, Yan-Ting

Shortliffe, Linda M. Dairiki

Shulman, Adrian

Shultz, Sandy

Shunya, Uchida

Sigvardsdotter, Erika

Silbert, Benjamin

Simka, Marian

Singh, Favil

Singh, Naveen

Sinjari, Bruna

Sisley, Stephanie

Sisti, Giovanni

Sitia, Giovanni

Sivapalan, Pradeesh

Skaug, Brian

Skinner, Jonathan R.

Skoien, Richard

Slankamenac, Ksenija

Smalling, Richard W.

Smith, Judith A.

Smith, Patrick

Söderström, Lisa

Soiza, Roy L.

Song, Yin-Chen

Sood, Manish M.

Sorensen, Carl

Soto-Pérez, Felipe

Spetea, Mariana

Spradley, Frank T.

Squires, Paul

Stančin, Sara

Stansfield, Kirstie

Starostik, Petr

Steiner, Laurie A.

Stepien, Karolina M.

Stodt, Benjamin

Stoffregen, Thomas

Stringhini, Silvia

Stunes, Astrid K.

Sturiale, Carmelo L.

Stylianou, Konstantinos

Sukhal, Shashvat

Sullivan, Mark D.
Sun, Zhong-Hua

Suto, Takahito

Suzuki, Takeshi

Svensson, Jannet

Szeliga, Jacek

Sztal, Tamar

Szuhany, Kristin L.

Tagliaferri, Sara

Takahashi, Yusuke

Takaya, Junji

Takebayashi, Shigeo

Takiyama, Yumi

Taniguchi, Hiroya

Tanner, Bobo

Tariq, Sarah

Tarragon Cros, Ernesto

Tedla, Yacob G.

Teede, Helena J.

Teles, Alisson

Tennant, Matthew T.S.

Teques, Pedro

Thamilselvan, Sivagnanam

Thevarajan, Irani

Thiersch, Markus

Thomas, Lucy C.

Thomas, Rebecca L.

Thompson, Wade

Thunnissen, Erik

Thuzar, Moe

Ticconi, Carlo

Tidbury, Laurence P.

Tieland, Michael

Tiller, Daniel

Timman, Reinier

Titus, Mark A.

Tjernberg, Ivar

Tokodai, Kazuaki

Toma, Claudio

Tonga, Katrina

Torgerson, Paul R.

Torres, Alcy R.

Tosur, Mustafa

Tõugu, Vello

Touraine, Philippe

Townsend, Eleanor

Trachana, Varvara

Treasure, Tom

Trimarco, Bruno

Trinkmann, Frederik

Trino, Stefania

Trummer, Olivia

Tsuruda, Toshihiro
Tunér, Jan

Turner, Raymond S.

Tvedt, Tor Henrik Anderson

Twomey, Niall

Ufholz, Kelsey E.

Ungaro, Paola

Valomon, Amandine

Van Ballegooijen, Hanne

Van Den Helder, Jantine

Van Hise, Nicholas W.

Van Rompay, Koen K.A.

Van Ryn, Joanne

Van Weerden, Wytske

Varga, Eszter

Vashisht, Rohit

Vassallo, Robert

Vassiliou, Aliki G.

Vassiliou, Leandros-Vassilios

Vaucher, Paul

Vaz, Paula

Vehkaoja, Antti

Verguts, Jasper

Vervoort, Tine

Vetter, Douglas E.

Vicent, Jesus

Viglianti, Elizabeth M.

Villani, Anthony

Villeneuve, Laurent

Vitacca, Michele

Vogel, Erin

Voges, Inga

Von Lewinski, Dirk

Vrana, David

Vukmirovic, Milica

Waalen, Jill

Wada, Tomohito

Wang, Henry E.

Wang, Peng-Hui

Wang, Shao-Meng

Warburton, Darren E.R.

Ward, Roberta J.

Wardle, Jon

Wargovich, Michael

Wasan, Ellen

Watanabe, Atsuyuki

Wedi, Edris

Weis, Sebastian

Wenning, Leslie

Werner, Gabriela G.

Westhoff-Bleck, Mechthild

Whelan, Fiona J.

White, Becky 
Wienbergen, Harm

Wiersinga, Willem J.

Wierzbicka, Elzbieta

Willis, Erin

Willis, Gareth R.

Wilsey, Michael

Wollin, Daniel

Wong, Yon-Cheong

Woroniecki, Robert P.

Wright, Eric

Wright, Laura

Wu, Chih-Jen

Wu, Tong-Zhi

Wu, Wen-Chih

Xiang, Hui-Yun

Xu, Da-Zhong

Xu, Jun-Wang
Xu, Qin-Zi
Xue, Xiang
Yamada, Tetsuji
Yamaoka, Yoshio
Yang, Eun Joo
Yang, Kwang Ik
Yang, Wei-Shan
Yang, Zheng-Yu
Yavuz, Metin
Yavuz, Sule
Yazawa, Takashi
Yébenes, Juan C.
Yeung, Eugene
Yevzlin, Alexander S.
Yin, Jun

Xu, Jun-Wang

Xue Xiang

Yamada, Tetsuji

Yamaoka, Yoshio

Yang, Eun Joo

Yang, Kwang Ik

Yang, Wei-Shan

Yang, Zheng-Yu

Yavuz, Metin

Yavuz, Sule

Yazawa, Takashi

Yébenes, Juan C.

Yevzlin, Alexander S.

Yin, Jun
Yokota, Tomoya

Yokoyama, Hisayo

$\mathrm{Yu}$, Guo-Qin

Yu, Min-Zhong

Yuan, Li-Yun

Yuzawa, Yukio

Zaccardi, Francesco

Zach, Sima

Zakaria, Dala

Zervos, Emmanuel E.

Zhang, Ling-Juan

Zhang, Meng-Liang

Zhang, Qiu-Yang

Zhao, Wei

Zoeller, Thomas

Zomer, Tizza P.

(C) 2019 by the author. Licensee MDPI, Basel, Switzerland. This article is an open access article distributed under the terms and conditions of the Creative Commons Attribution (CC BY) license (http://creativecommons.org/licenses/by/4.0/). 